\title{
Moments of pion distribution amplitude using operator product expansion on the lattice
}

\author{
William Detmold \\ Centre for Theoretical Physics, Massachusetts Institute of Technology, MA 02139, USA \\ E-mail: wdetmoldemit.edu \\ Issaku Kanamori \\ Department of Physical Science, Hiroshima University, Higashi-Hiroshima 739-8526, Japan \\ E-mail: kanamori@hiroshima-u.ac.jp \\ C.-J. David Lin \\ Institute of Physics, National Chiao-Tung University, Hsinchu 30010, Taiwan \\ Centre for High Energy Physics, Chung-Yuan Christian University, Chung-Li, 32032, Taiwan \\ E-mail: dlin@mail.nctu.edu.tw
}

\section{Santanu Mondal ${ }^{*}$}

Institute of Physics, National Chiao-Tung University, Hsinchu 30010, Taiwan

E-mail: santanu.sinp@gmail.com

\section{Yong Zhao}

Centre for Theoretical Physics, Massachusetts Institute of Technology, MA 02139, USA

E-mail: yzhaoqca@mit.edu

\begin{abstract}
We report an exploratory study of the current-current matrix elements that are relevant to the extraction of moments of the pion light-cone distribution amplitude, employing the method of introducing a valence relativistic heavy quark. The numerical investigation is carried out in the quenched approximation with the physical volume $L \approx 2.4 \mathrm{fm}$ at two values of lattice spacing $(0.05$ and $0.075 \mathrm{fm})$. We obtain clean signals for the relevant Euclidean hadronic tensor with reasonable statistics, but observe that the lattice artefacts are non-negligible in our results. The key conclusion from the analysis hitherto is that although our approach has the potential for making significant contributions to parton physics, data at finer lattice spacings that are currently being produced are needed in order to control the continuum extrapolation.
\end{abstract}

The 36th Annual International Symposium on Lattice Field Theory - LATTICE2018

22-28 July, 2018

Michigan State University, East Lansing, Michigan, USA.

\footnotetext{
*Speaker.
} 


\section{Introduction}

Numerical implementation of lattice field theory is normally carried out in Euclidean space. Therefore it is challenging to apply lattice QCD to extract reliable results in parton physics, which involves non-perturbative dynamics on the light cone. For this reason, the traditional approach to extracting various parton distribution functions (PDF's) and light-cone distribution amplitudes (LCDA's) employing lattice QCD relies on the calculation of Mellin moments. These moments are related to matrix elements of local operators that arise from an operator product expansion (OPE). Because $\mathrm{O}(4)$ Euclidean space-time symmetry is broken by the lattice geometry, these local operators mix under renormalisation, resulting in power divergences that are difficult to subtract accurately $[1,2]$. This is the reason why lattice-QCD determination of the PDF's and the LCDA's using the above strategy has only been giving results for the first three Mellin moments.

Alternative methods for gaining parton-physics information with lattice QCD have been suggested over the past two decades $[3,4,5,6,7,8,9,10,11]$. These methods involve calculating hadronic matrix elements of non-local operators, and many of them are presently under intensive investigation [12]. In this article, we present progress of performing a lattice calculation for the pion LCDA, employing the proposal of introducing a valence relativistic heavy quark, as detailed for the quark PDF's in Ref. [5]. The pion LCDA, $\phi_{\pi}(\xi)$, is of importance in understanding hadronic exclusive decay processes in QCD [13], as well as in extracting information in flavour physics [14]. It is defined as

$$
\left\langle 0\left|\bar{d}(-z) \gamma_{\mu} \gamma_{5} \mathscr{W}[-z, z] u(z)\right| \pi^{+}(p)\right\rangle=i p_{\mu} f_{\pi} \int_{-1}^{1} d \xi \mathrm{e}^{-i \xi p \cdot z} \phi_{\pi}(\xi)
$$

with $z^{2}=0$, and $\mathscr{W}[-z, z]$ being a light-like Wilson line between $-z$ and $z$. The variable $\xi$ represents the fraction of the pion momentum carried by the valence $u$ quark. The above DA can be constructed from its Mellin moments, $a_{n}$, that are related to local matrix elements in QCD,

$$
\begin{aligned}
& a_{n}=\int_{0}^{1} d \xi \xi^{n} \phi_{\pi}(\xi), \\
& f_{\pi} a_{n-1}\left[p^{\mu_{1}} \ldots p^{\mu_{n}}-\text { Traces }\right]=-i\left\langle 0\left|\bar{d} \gamma^{\left\{\mu_{1}\right.} \gamma^{5}\left(i D^{\mu_{2}}\right) \ldots\left(i D^{\left.\mu_{n}\right\}}\right) u-\operatorname{Traces}\right| \pi^{+}(p)\right\rangle,
\end{aligned}
$$

with the Lorentz indices symmetrised. From early [2] to recent $[15,16,17,18]$ lattice calculations following the traditional approach, only the second moment of this LCDA has been extracted because of the above issue of power divergence in the operator mixing. Using the strategies in Refs. [6,8], exploratory results for the $\xi$-dependence of $\phi_{\pi}(\xi)$ have recently been reported $[19,20]$.

\section{Operator product expansion and the valence heavy quark}

Using the method of Ref. [5], it can be shown that the Euclidean hadronic tensor,

$$
U_{A}^{[\mu v]}(q, p)=\int d^{4} x e^{i q x}\left\langle 0\left|T\left[A_{\Psi, \psi}^{[\mu}(x) A_{\Psi, \psi}^{v]}(0)\right]\right| \pi^{+}(p)\right\rangle,
$$

in the continuum limit enables one to extract the moments, $a_{n}$, defined in Eq. (1.2) without having to subtract any power divergence. In Eq. (2.1), the Lorentz indices, $\mu$ and $v$, are antisymmetrised, 
and the axial current is defined as

$$
A_{\Psi, \psi}^{\mu}=\bar{\Psi} \gamma^{\mu} \gamma_{5} \psi+\bar{\psi} \gamma^{\mu} \gamma_{5} \Psi
$$

with $\psi$ being a light-quark and $\Psi$ being the valence heavy-quark fields. We stress that the approach outlined in Ref. [5] requires the extrapolation of lattice results for $U_{A}^{[\mu v]}(q, p)$ to the continuum limit. Furthermore, the hadronic tensor, $U_{A}^{[\mu v]}(q, p)$, should be computed in the "unphysical" regime

$$
\left(p_{M}+q_{M}\right)^{2}<\left(m_{\Psi}+\Lambda_{\mathrm{QCD}}\right)^{2},
$$

where $q_{M}$ and $p_{M}$ are the Minkowskian counterparts of $q$ and $p$. In this regime the analytic continuation of $U_{A}^{[\mu v]}(q, p)$ to Minkowski space is straightforward. It can be achieved by simply relating $q_{4}$ to $i q_{0}$. The above considerations lead to the requirement for the hierarchy of scales

$$
\Lambda_{\mathrm{QCD}} \ll \sqrt{q^{2}} \lesssim m \Psi \ll \frac{1}{a},
$$

where $a$ is the lattice spacing.

Performing an OPE by following the procedure in Ref. [5], one obtains

$$
U_{\tilde{A}}^{[\mu v]}(p, q)=2 i f_{\pi} \varepsilon_{\mu v \rho \lambda} q^{\rho} p^{\lambda} \times \sum_{n=0,2,4 \cdots}^{\infty}\left[\frac{\zeta^{n} C_{n}^{2}(\eta)}{(n+1) \tilde{Q}^{2}}\right] \mathscr{C}_{W}^{(n)}\left(m_{\Psi}, \tilde{Q}, \mu\right) a_{n}(\mu)
$$

where $\zeta=\sqrt{p^{2} q^{2}} / \tilde{Q}^{2}, \eta=p \cdot q / \sqrt{p^{2} q^{2}}, \tilde{Q}^{2}=-q^{2}-m_{\Psi}^{2}$, the $\mathscr{C}_{W}^{(n)}\left(m_{\Psi}, \tilde{Q}, \mu\right)$ are the Wilson coefficients, $\mu$ is the renormalisation scale, and the $C_{n}^{2}(\eta)$ are the Gegenbauer polynomials that arise from resumming the target-mass effects. Notice that there is an ambiguity of $O\left(\Lambda_{\mathrm{QCD}}\right)$ in the definition of $\tilde{Q}$. Detailed discussion of this ambiguity and the higher-twist contributions can be found in Ref. [5]. Also, Eq. (2.5) indicates that the hadronic tensor, $U_{A}^{[\mu v]}(p, q)$, is purely imaginary in Euclidean space.

\section{Correlators and simulation details}

We consider the following correlators involving the pion interpolating operator, $\mathscr{O}_{\pi}$, and the current in Eq. (2.2),

$$
\begin{aligned}
C_{3}^{\mu v}\left(\tau_{e}, \tau_{s} ; \vec{p}_{e}, \vec{p}_{s}\right) & =\int d^{3} x_{e} d^{3} x_{s} \mathrm{e}^{i \vec{p}_{e} \cdot \vec{x}_{e}+i \vec{p}_{s} \cdot \vec{x}_{s}}\left\langle 0\left|\mathrm{~T}\left[A_{\Psi, \psi}^{\mu}\left(\vec{x}_{e}, \tau_{e}\right) A_{\Psi, \psi}^{v}\left(\vec{x}_{m}, \tau_{s}\right) \mathscr{O}_{\pi}^{\dagger}(0)\right]\right| 0\right\rangle \\
C_{\pi}\left(\tau_{\pi} ; \vec{p}\right) & =\int d^{3} x \mathrm{e}^{i \vec{p} \cdot \vec{x}}\left\langle 0\left|\mathscr{O}_{\pi}\left(\vec{x}, \tau_{\pi}\right) \mathscr{O}_{\pi}^{\dagger}(0)\right| 0\right\rangle
\end{aligned}
$$

where the subscripts, $e$ and $s$, in the three-point function stand for "extended" and "sink" points in the computation of the quark propagators. It is straightforward to demonstrate that using $\mathrm{C}_{3}^{\mu v}$ and $\mathrm{C}_{\pi}$ in the limit where $\tau_{\pi, e, s}$ are all large, one can extract the quantity, $R_{3}^{\mu v}$, that is defined as

$$
R_{3}^{\mu v}(\tau, \vec{q}, \vec{p}) \equiv \int d^{3} x e^{i \vec{q} \cdot \vec{x}}\left\langle 0\left|T\left[A_{\Psi, \psi}^{\mu}(\vec{x}, \tau) A_{\Psi, \psi}^{v}(\overrightarrow{0}, 0)\right]\right| \pi(p)\right\rangle .
$$

Thus the hadronic tensor in Eq. (2.1) can be obtained by performing the Fourier transform of $R_{3}^{[\mu \nu]}(\tau, \vec{q}, \vec{p})$ in the temporal direction,

$$
U_{A}^{[\mu v]}(q, p)=\int_{\tau_{\min }}^{\tau_{\max }} d \tau e^{i q_{4} \tau} R_{3}^{[\mu v]}(\tau, \vec{q}, \vec{p})
$$



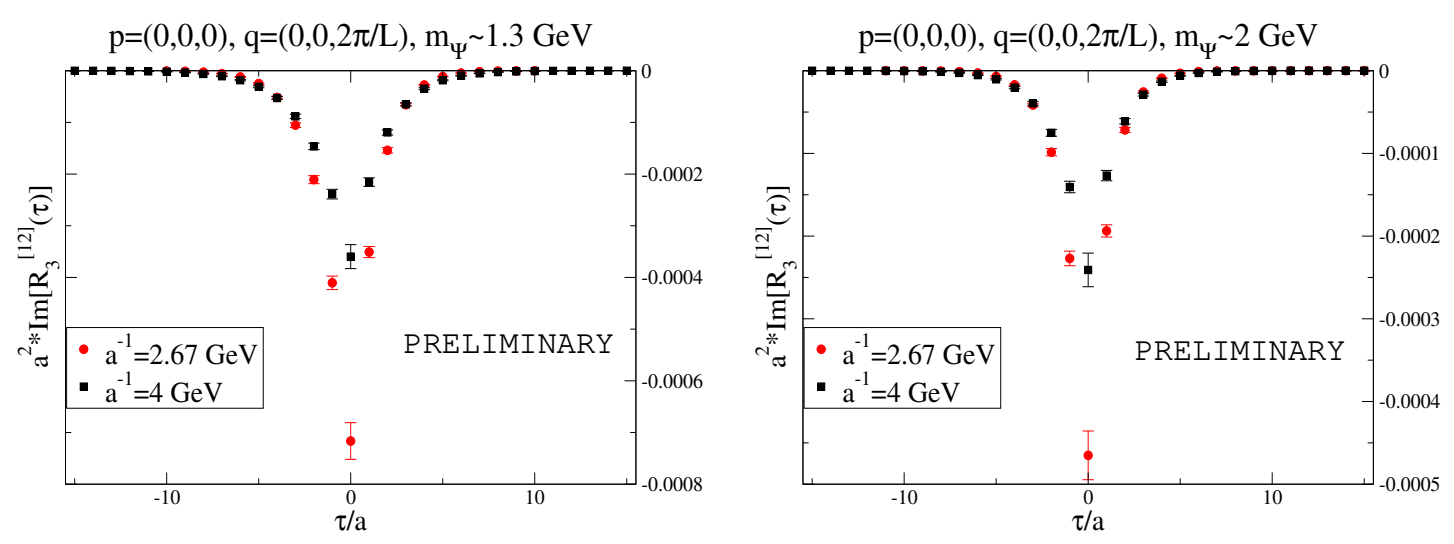

Figure 1: Imaginary part of bare $R_{3}^{[12]}(\tau, \vec{q}, \vec{p})$ with $\vec{q}=(0,0,2 \pi / L)$ and $\vec{p}=(0,0,0)$, at $m_{\Psi}=1.3 \mathrm{GeV}$ (left) and $m_{\Psi}=2 \mathrm{GeV}$ (right).

where the integration range, $\left[\tau_{\min }, \tau_{\max }\right]$, is constrained by the range of Euclidean time where we are able to isolate the one-pion state in $\mathrm{C}_{3}^{\mu \nu}$ and $\mathrm{C}_{\pi}$. Because of the use of the valence heavy quark, which exponentially localises the signal, we find that the above Fourier transform is well approximated when $\tau_{\min } \sim-0.7 \mathrm{fm}$ and $\tau_{\max } \sim 0.7 \mathrm{fm}$ for the results reported here.

Our simulations are performed in the quenched approximation with Wilson gauge action. Gauge field ensembles are generated at several values of lattice spacing, $a$, and the finest lattice is at $a^{-1}=8 \mathrm{GeV}^{1}$. All these ensembles are tuned to have the same spatial lattice size, $L \approx 2.4$ $\mathrm{fm}$, with the temporal extent being $2 L$. Valence fermion propagators are computed employing non-perturbatively $O(a)$-improved Wilson action, with values of the clover coefficient determined using the result of Ref. [22]. Since this is an exploratory investigation, we use the one-loop matching coefficient, $Z_{A}$, for the axial current in Eq. (2.2) [24], without $O(a)$-improving it. However, the quark-field normalisation proposed in Ref. [23] is employed to reduce lattice artefacts.

At this conference, our presentation focuses on calculations from two lattice spacings, $a=$ $0.075 \mathrm{fm}\left(a^{-1}=2.67 \mathrm{GeV}\right)$ and $a=0.05 \mathrm{fm}\left(a^{-1}=4 \mathrm{GeV}\right)$, and at two choices of heavy-quark masses, $m_{\Psi}=1.3 \mathrm{GeV}$ and $2 \mathrm{GeV}^{2}$. The input bare light-quark mass is tuned such that the pion mass $M_{\pi}=450 \mathrm{MeV}$ in this exploratory study.

\section{Exploratory results}

Figure 1 shows results for the imaginary part of bare $R_{3}^{[12]}(\tau, \vec{q}, \vec{p})$ with $\vec{q}=(0,0,2 \pi / L)$ and $\vec{p}=(0,0,0)$, at $m_{\Psi}=1.3 \mathrm{GeV}$ and $2 \mathrm{GeV}$. The temporal-direction Fourier transforms [Eq. (3.3)] on these ratios for obtaining the corresponding hadronic tensors are displayed in Fig. 2, where we are showing the cases of $q_{4}=0$ and $q_{4}=1.2 i \mathrm{GeV}$. For this choice of the momenta, result of the OPE for the hadronic tensor is significantly simplified, and in principle allows us to extract the moments, $\left\{a_{n}\right\}$, through varying $q_{4}$. However, we do not attempt such numerical exercise at this stage, because the lattice artefacts are observed to be non-negligible, as evidenced in Figs. 2 and

\footnotetext{
${ }^{1}$ Ensembles with $a^{-1}>2.7 \mathrm{GeV}$ are generated by employing the method proposed in Ref. [21].

${ }^{2}$ We also have exploratory results at $a=0.06 \mathrm{fm}\left(a^{-1}=3.33 \mathrm{GeV}\right)$ for $m \Psi=1.3 \mathrm{GeV}$. They are included in Fig. 3.
} 

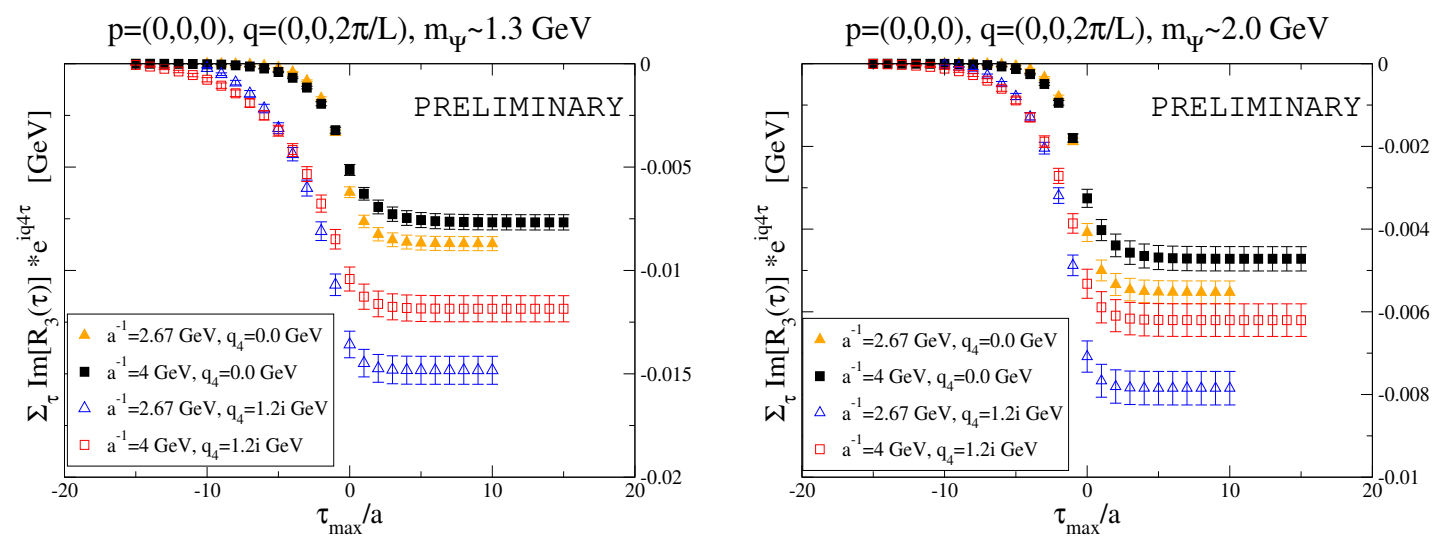

Figure 2: Time-direction Fourier transform for obtaining bare $\operatorname{Im}\left[U_{A}^{[12]}(q, p)\right]$ with $\vec{q}=(0,0,2 \pi / L)$ and $\vec{p}=(0,0,0)$ and two choices $q_{4}$, at $m_{\Psi}=1.3 \mathrm{GeV}$ (left) and $2 \mathrm{GeV}$ (right).

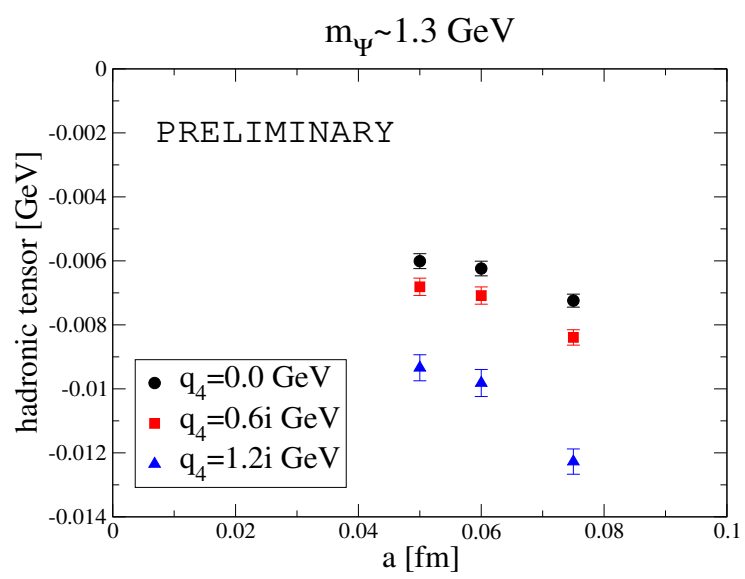

Figure 3: Imaginary part of $U_{A}^{[12]}(q, p)$ for $\vec{q}=(0,0,2 \pi / L), \vec{p}=(0,0,0)$ and $m_{\Psi}=1.3 \mathrm{GeV}$, at $a=0.05$, $0.06,0.075 \mathrm{fm}\left(a^{-1}=4.0,3.33,2.67 \mathrm{GeV}\right.$, respectively), with 3 choices of $q_{4}$. One-loop $Z_{A}$ is used.

3. Notice that we also include the exploratory results from the $a=0.06 \mathrm{fm}$ lattice in Fig. 3. As stressed earlier in this article, our strategy requires reliable extrapolation of the hadronic tensor to the continuum limit.

We have also examined $R_{3}^{[12]}(\tau, \vec{q}, \vec{p})$ and $U_{A}^{[12]}(p, q)$ at non-vanishing pion momentum. In this case, the lattice data are expected to be noisy. To address this, we have investigated the technique of momentum smearing [25] for the pion interpolating operator, $\mathscr{O}_{\pi}$. Results of the study for two choices of the pion momentum, $\vec{p}=(0,0,2 \pi / L)$ and $\vec{p}=(0,2 \pi / L, 2 \pi / L)$, at the current-injected momentum $\vec{q}=(0,0,2 \pi / L)$ are presented in Figs. 4 and 5. Plots in these figures show that momentum smearing is advantageous already for these low values of $|\vec{p}|$, although its implementation requires separate computations for the light quark and anti-quark propagators. We will make use of this technique in our future work on the pion LCDA. 

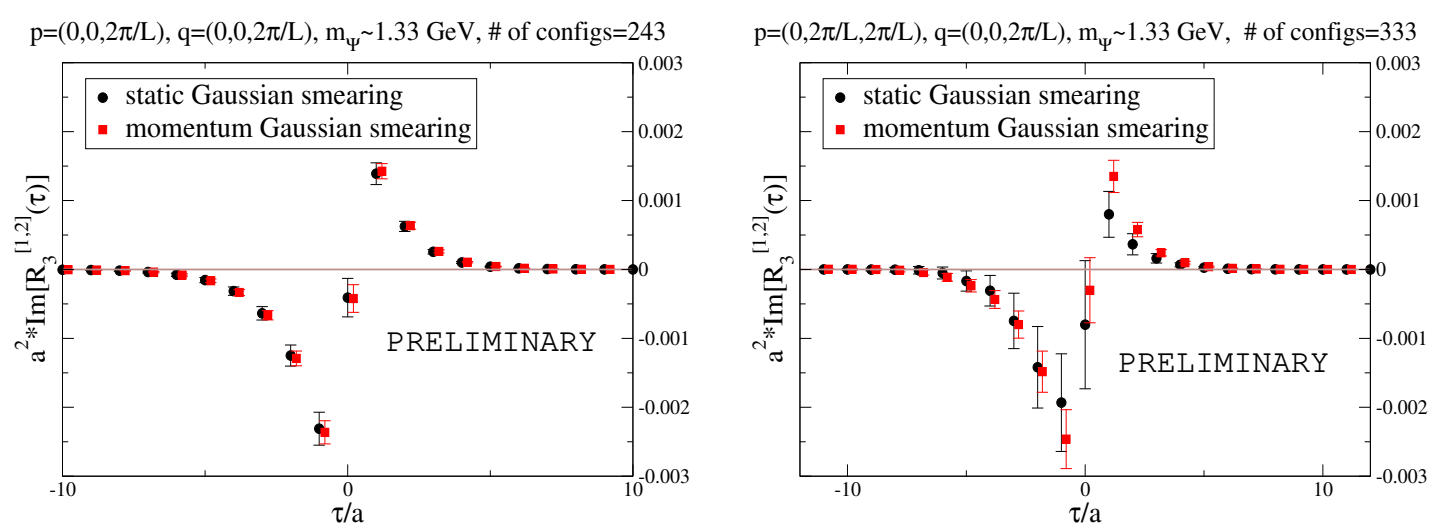

Figure 4: Bare $R_{3}^{[12]}(\tau, \vec{q}, \vec{p})$ with $\vec{q}=(0,0,2 \pi / L)$ and $m_{\Psi}=1.3 \mathrm{GeV}$ at $\vec{p}=(0,0,2 \pi / L)$ (left) and $\vec{p}=$ $(0,2 \pi / L, 2 \pi / L)$ (right). Black circle is for the usual gauge invariant Gaussian source and sink, red square is for the momentum smearing with momentum $\pm 0.7 \vec{p}$ for quark and anti-quark.
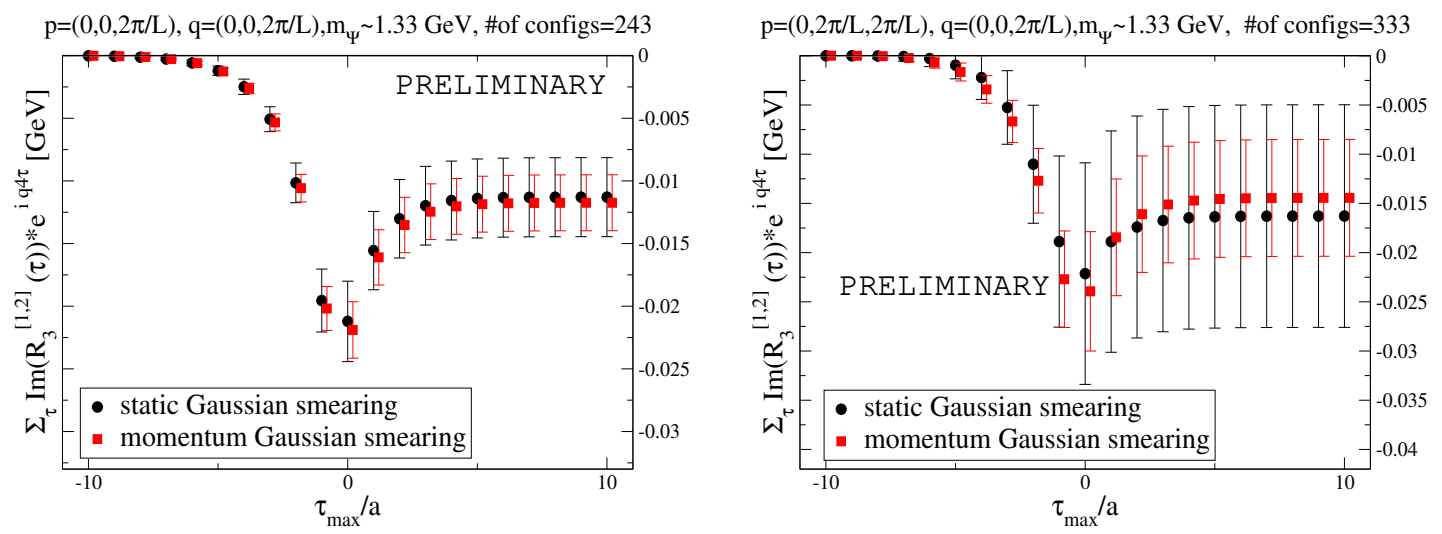

Figure 5: Bare $\operatorname{Im}\left[U_{A}^{[12]}(q, p)\right]$ with $\vec{q}=(0,0,2 \pi / L)$ and $m \Psi=1.3 \mathrm{GeV}$ at $\vec{p}=(0,0,2 \pi / L)$ (left) and $\vec{p}=$ $(0,2 \pi / L, 2 \pi / L)$ (right). Black circle is for the usual gauge invariant Gaussian source and sink, red square is for the momentum smearing with momentum $\pm 0.7 \vec{p}$ for quark and anti-quark.

\section{Conclusion and outlook}

In this article, we report progress of our exploratory investigation of the pion LCDA using the strategy of introducing a valence heavy quark in the current-current correlator. This strategy allows us to extract higher moments for this LCDA. We find that reasonably good signals can be obtained for the relevant Euclidean hadronic tensor. Although this demonstrates a promising future of this approach, we observe that lattice artefacts can still be non-negligible in the regime $0.05 \mathrm{fm} \lesssim a \lesssim$ $0.075 \mathrm{fm}$. Since our method requires reliable continuum extrapolation, it is necessary to have data at finer lattice spacings for this task, as will be studied in the near future.

\section{Acknowledgments}

We grateful to Michael Endres for generating the gauge configurations, and to Balint Joo for 
helping us with the QPhiX library. WD and YZ are supported by the U.S. Department of Energy, Office of Science, Office of Nuclear Physics, from de-sc0011090 and within the framework of the TMD Topical Collaboration, and thank National Chiao-Tung University for hospitality. WD is also supported by the U.S. Department of Energy under Early Career Research Award de-sc0010495 and the SciDAC4 award de-sc0018121. IK acknowledges Priority Issue 9 to be tacked by Using Post K Computer and Joint Institute for Computational Science (JICFuS). CJDL and SM are supported by Taiwanese MoST via grants 105-2112-M-002-023-MY3, and thank MIT for hospitality. We acknowledge support by the MIT MISTI program.

\section{References}

[1] A. S. Kronfeld and D. M. Photiadis, Phys. Rev. D 31 (1985) 2939.

[2] G. Martinelli and C. T. Sachrajda, Phys. Lett. B 190 (1987) 151.

[3] U. Aglietti et al., Phys. Lett. B 441 (1998) 371 [hep-ph/9806277].

[4] K. F. Liu, Phys. Rev. D 62 (2000) 074501 [hep-ph/9910306].

[5] W. Detmold and C. J. D. Lin, Phys. Rev. D 73 (2006) 014501 [hep-lat/0507007].

[6] V. Braun and D. Mueller, Eur. Phys. J. C 55 (2008) 349 [arXiv:0709.1348 [hep-ph]].

[7] Z. Davoudi and M. J. Savage, Phys. Rev. D 86 (2012) 054505 [arXiv:1204.4146 [hep-lat]].

[8] X. Ji, Phys. Rev. Lett. 110 (2013) 262002 [arXiv:1305.1539 [hep-ph]].

[9] A. J. Chambers et al., Phys. Rev. Lett. 118 (2017) no.24, 242001 [arXiv:1703.01153 [hep-lat]].

[10] A. V. Radyushkin, Phys. Rev. D 96 (2017) no.3, 034025 [arXiv:1705.01488 [hep-ph]].

[11] Y. Q. Ma and J. W. Qiu, Phys. Rev. Lett. 120 (2018) no.2, 022003 [arXiv:1709.03018 [hep-ph]].

[12] C. J. Monahan, these proceedings.

[13] G. P. Lepage and S. J. Brodsky, Phys. Rev. D 22 (1980) 2157.

[14] M. Beneke et al., Phys. Rev. Lett. 83 (1999) 1914 [hep-ph/9905312].

[15] V. M. Braun et al., Phys. Rev. D 74 (2006) 074501 [hep-lat/0606012].

[16] R. Arthur et al., Phys. Rev. D 83 (2011) 074505 [arXiv:1011.5906 [hep-lat]].

[17] V. M. Braun et al., Phys. Rev. D 92 (2015) no.1, 014504 [arXiv:1503.03656 [hep-lat]].

[18] G. S. Bali et al. [RQCD Collaboration], Phys. Lett. B 774 (2017) 91 [arXiv:1705.10236 [hep-lat]].

[19] J. H. Zhang et al., Phys. Rev. D 95 (2017) no.9, 094514 [arXiv:1702.00008 [hep-lat]].

[20] G. S. Bali et al., arXiv:1807.06671 [hep-lat].

[21] M. G. Endres et al., Phys. Rev. D 92 (2015) no.11, 114516 [arXiv:1510.04675 [hep-lat]].

[22] R. G. Edwards, U. M. Heller and T. R. Klassen, Phys. Rev. Lett. 80 (1998) 3448 [hep-lat/9711052].

[23] A. X. El-Khadra it et al., Phys. Rev. D 55 (1997) 3933 [hep-lat/9604004].

[24] E. Gabrielli et al., Nucl. Phys. B 362 (1991) 475.

[25] G. S. Bali et al., Phys. Rev. D 93 (2016) no.9, 094515 [arXiv:1602.05525 [hep-lat]]. 